\section{La lectura de}

\section{textos académicos}

en la formación

universitaria de docentes:

\section{Entre supuestos y estrategias}

\section{Reading academic texts in the teacher's undergraduate education:

\author{
Between suppositions and \\ strategies
}

\section{A leitura de textos acadêmicos na formação universitária de professores: Cenários e estratégias}

Lilia Cañón Flórez*

Jennyfer Mancera Baquero**

Nataly Ruiz Rojas

* Magíster en Lingüística Española. Profesora Facultad de Educación Universidad Pedagógica Nacional, Universidad de la Salle. Correo electrónico: lili.canon@hotmail.com

** Licenciada en Psicología y Pedagogía, Universidad Pedagógica Nacional. Monitora de investigación, CIUP, 2009. Correo electrónico: ferdemanzana@hotmail.com

*** Licenciada en Psicología y Pedagogía, Universidad Pedagógica Nacional. Monitora de investigación, CIUP, 2009. Correo electrónico: nanadeoso@gmail.com

El artículo es el resultado del proyecto de investigación "Estrategias cognitivas y metacognitivas de comprensión lectora de los textos académicos en el ámbito universitario" que se adelantó entre febrero de 2009 y mayo de 2010 y contó con el apoyo de la Facultad de Educación y del Centro de Investigaciones de la Universidad Pedagógica Nacional (CIUP).

\section{Resumen}

El presente artículo presenta los hallazgos de un estudio en el que se indaga por las estrategias cognitivas y metacognitivas que propician los docentes y a las que recurren los estudiantes para adentrase en las exigencias de comprensión lectora de los textos académicos. El estudio fue realizado con estudiantes y profesores de la Facultad de Educación de la Universidad Pedagógica Nacional de Colombia.

\section{Palabras clave}

Comprensión lectora, texto académico, formación docente, ámbito universitario.

\section{Abstract}

This article presents the finds of a research study in which makes inquiries about cognitive and meta-cognitive strategies that are fostered by university teachers and the ones students turn to go deeper in the demands of reading comprehension of academic texts. This study was done with students and teachers of the School of Education of Universidad Pedagógica Nacional (National Pedagogic University) in Colombia.

\section{Key words}

Reading comprehension, academic text, teacher education, university field.

\section{Resumo}

Este artigo apresenta os resultados de um estudo que investiga as estratégias cognitivas e metacognitivas propostas pelos professores, às que os alunos recorrem para equiparar-se às demandas de compreensão de leitura de textos acadêmicos. 0 estudo foi realizado com os alunos e professores da Faculdade de Educação da Universidade Pedagógica Nacional da Colômbia.

\section{Palavras-chave}

Compreensão leitora, texto acadêmico, formação de professores, campo universitário.

Fecha de recepción: 30 de julio de 2010 Fecha de aprobación: 28 de octubre de 2010 ................................................... 


\section{Del problema}

E el ámbito universitario la lectura se presume esencial tanto para el aprendizaje como para las respuestas a la exigencia académica. Por demás, la lectura se categoriza como medio fundamental para la adquisición de información, para el aprendizaje y como acercamiento a procesos de formación. En consecuencia, comprender textos se convierte en tarea básica en la educación universitaria.

Ahora bien, en términos generales, se considera que los estudiantes en su formación básica van construyendo los procesos de lectura que les permitirían enfrentarse de manera adecuada a las exigencias y a las necesidades del ámbito universitario; por ende, se espera que se encuentren en capacidad de enfrentar por su cuenta textos académicos. Asimismo, en este nivel de escolaridad, los profesores suponen el manejo de niveles básicos de estrategias de lectura. Por ello quedaría de su parte propiciar mecanismos que incentiven y fortalezcan la lectura autónoma de tales textos.

Sin embargo, se ha encontrado que gran cantidad de estudiantes evidencian algunos problemas y dificultades en la comprensión de textos. A su vez, y a pesar de que se convoca desde las políticas y desde los modelos didácticos en boga a un trabajo interdisciplinario, el quehacer docente en no pocos casos se centra en "parcelas de conocimiento" y se delega a los "especialistas" la preocupación por evaluar y transformar las estrategias de trabajo con el texto académico.

En este ámbito pareciera que los intereses de los docentes, e incluso de los estudiantes, se centraran en los resultados y, por tanto, en las formas y en los mecanismos de evaluación más que en los procesos. De ahí que, frente las trabas en la formación profesional, como resultado de los "problemas" de lectura, se opte por la teoría del déficit. Desde esta perspectiva, se torna común encontrar vías de solución centradas en resolver supuestos problemas de comprensión lectora para llegar a resultados específicos. En términos de medicina se diría que se está atendiendo la enfermedad y no al enfermo.

No obstante, se hace necesario reconocer que, desde la concepción de la lectura como proceso interactivo y transaccional, en la formación universitaria no solo se debe atender el déficit del lector frente al texto ni limitarse a los productos de la lectura: se han de considerar las formas como el estudiante universitario y los procesos académicos que lo acompañan contribuyen a generar estrategias de comprensión. Por tal razón, se considera pertinente preguntar por las estrategias cognitivas y metacognitivas que propician los docentes y a las que recurren los estudiantes para adentrase en las exigencias de comprensión lectora de los textos académicos en un ámbito particular como el de los pregrados de la Facultad de Educación de la Universidad Pedagógica Nacional.

\section{De las aproximaciones teóricas}

La lectura como mediación. Reconocer, desde la concepción de la lectura como proceso interactivo y transaccional, las formas como el estudiante universitario, lector adulto, y los procesos académicos que lo acompañan generan estrategias de comprensión lectora implica: asumir la "decodificación" como un medio y no como un fin y la lectura como un "proceso de creación de significados", ubicar el objeto de estudio en las estrategias y no propiamente en los déficits de comprensión; y valorar tanto las estrategias que sugieren los maestros como las que usan los estudiantes.

Ahora, si se acepta que el texto determina solo en parte su propio significado, también se ha de reconocer que el lector forja intencionalmente el sentido; es decir, del lector y de lo que aporta al proceso depende en gran medida la construcción del significado del texto. Así, la lectura se convierte en un evento interactivo, intencional, constructivo e inferencial caracterizado por la formación y la comprobación permanentes de hipótesis acerca del contenido del texto (Goodman, 1967; Smith, 1971).

“... desde la concepción de la lectura como proceso interactivo y transaccional, en la formación universitaria no solo se debe atender el déficit del lector frente al texto ni limitarse a los productos de la lectura: se han de considerar las formas como el estudiante universitario y los procesos académicos que lo acompañan contribuyen a generar estrategias de comprensión." 
“...si se acepta que el texto

determina solo en parte su propio significado, también se ha de reconocer que el lector forja intencionalmente el sentido; es decir, del lector y de lo que aporta al proceso depende en gran medida la construcción del significado del texto. Así, la lectura se convierte en un evento interactivo, intencional, constructivo e inferencial..."

El anterior concepto implica reconocer un lector intencional, autónomo, independiente y responsable de su propio aprendizaje; y en la medida que el lector sea autónomo, el acto de leer supone un pensamiento reflexivo, analítico y crítico: un leer para aprender, en términos de Beaugrande (1984). En concordancia, la comprensión de lectura se ha de reconocer como un proceso gradual y estratégico de creación de sentido a partir de la interacción del lector con el texto en un contexto particular. Dicha interacción permite al lector involucrarse en procesos de inferencia necesarios para construir y revisar sus representaciones de lo expuesto en el texto. La lectura, en consecuencia, se constituye en un proceso de inferencias que permiten al lector llevar a cabo operaciones cognitivas en su intento por construir un significado del texto y por elaborar una representación coherente de los contenidos (González, 1991).

En esta perspectiva, la claridad que tenga un lector en relación con el papel de las mediaciones y de los procesos cognitivos al momento de leer le permite regular sus procesos de lectura; en otros términos, llegaría a ser consciente de la secuencia de actividades intencionales y deliberadas a las cuales acude para lograr las metas que se ha propuesto. De esta manera, se pasa a niveles más formales de cognición, o sea, a la metacognición; ésta implica el alcance, por parte de un lector, de un estado desde el cual pueda controlar y regular de manera consciente sus procesos de construcción de sentido.

Metacognición. El concepto de metacognición se ha relacionado con memoria, atención, solución de problemas, estrategias, comprensión del lenguaje y aprendizaje (López y Arciniegas, 2004); en fin, se relaciona con -o mejor, parte de- la cognición. Si la actividad cognitiva implica percibir, comprender, aprender, recordar y pensar (Mateos, 2001, p.19), entonces, cuando se pretenda llevar tales procesos a determinadas tareas se requiere una actividad intelectual que actúe en tal dominio: se apela a la metacognición. En consecuencia, si lo cognitivo se relaciona con el conocimiento, lo metacognitivo se refiere al hecho de tener conciencia de tal conocimiento y de cómo se logra. En otras palabras, saber qué se sabe y no se sabe, así como también qué se conoce acerca del propio sistema cognitivo: capacidades y limitaciones y, por tanto, aquello que se ha de realizar para llegar a saber (Flavell, 1981, en Mateos, 2001; Baker y Brown, 1984).

Etapas de la metacognición. Con el fin de atender la consecución de los objetivos básicos de la toma de conciencia de la lectura como proceso estratégico y de sus implicaciones en el proceso de comprensión y en el autocontrol y la autorregulación de todo el proceso, distintos autores han propuesto tres etapas para poner en práctica las estrategias metacognitivas:

Planificación. Precisar los objetivos y las metas de lectura, los conocimientos en relación con el tema, el plan de acción y las estrategias por usar. Supervisión. Comprobar si se está llevando a cabo lo planificado; verificar la eficacia y la adecuación de las estrategias a los objetivos; si se encuentran dificultades, determinar a qué obedecen y cómo se pueden resolver. Evaluación tanto de los procesos desarrollados durante la lectura como de los resultados obtenidos. Supone no sólo constatar el nivel de comprensión que se va alcanzando sino, además, evaluar durante todo el proceso, las distintas operaciones que constituyen la actividad lectora.

De acuerdo con lo planteado hasta ahora, para lograr un proceso realmente eficaz, reflexivo y crítico de lectura para aprender, se hace necesario que el lector asuma el control, la supervisión y la evaluación permanentes de su propio proceso de comprensión. Desde tal perspectiva, se ha de señalar que si el estudiante universitario asume la lectura como proceso para aprender, si hace uso de estrategias que le permitan el logro de conocimiento, de saber, reconocerá la necesidad de supervisar y regular permanentemente su propio proceso. Por su parte, el docente, además de asignar lecturas específicas, propondrá tareas que hagan evidente el uso de tales estrategias para acompañar procesos eficaces. 


\section{De la metodología}

Indagar acerca de las estrategias cognitivas y metacognitivas en la comprensión de textos va más allá del reconocimiento de los propios procesos lectores y de las repercusiones en el actuar como estudiante universitario: da lugar a que, a partir de conocerse y reconocerse como lector, se abran caminos que recaerán en las futuras formas de encaminar actos de enseñanza de las nuevas generaciones de maestros.

En este contexto, el proyecto de investigación "Estrategias cognitivas y metacognitivas de comprensión lectora de los textos académicos en el ámbito universitario" reconoció desde un principio que el problema no se ubica en la deficiencia; radica en los procesos de construcción de sentido. De ahí que estimó como objetivo principal identificar y valorar las estrategias cognitivas y metacognitivas que propician los docentes y a las que recurren los estudiantes para adentrase en las exigencias de comprensión lectora de los textos académicos en el ámbito universitario. Para ello, se tendrían que cumplir dos objetivos específicos: identificar y valorar, en primera instancia, las estrategias cognitivas y metacognitivas que propician los docentes para que los estudiantes se adentren en las exigencias de
"Desde el punto de vista metodológico el proyecto se estructuró a partir de los planteamientos de la etnografía educativa como una disciplina que, en este caso particular, procura caminos para hacer evidente la forma como un grupo de estudiantes de la Facultad de Educación asume el control, la supervisión y la evaluación permanentes de su propio proceso de comprensión de textos académicos.”
La fase descriptiva del proyecto pretendió un acerperiversos instrumentos: una encuesta dadas realizadas por los profesores; un diario de campo a partir de la observación no participante de clases 列 por los estudiantes.

Los instrumentos de recolección de información. se estructuraron, como primer paso, dos encuestas para hacer visibles las directrices y las acciones que se desarrollan antes, durante y después de la lectura de textos académicos.

La encuesta dirigida a los estudiantes, una vez reconfigurada tras las pruebas piloto, se organizó en torno a 16 preguntas que pretendían acercarse, por un lado, a las directrices para asumir los procesos de lectura y a las pautas de evaluación de la comprensión formuladas por los docentes y, de otra parte, a las estrategias utilizadas por los estudiantes. La encuesta aplicada a los docentes también incluyó 16 preguntas para obtener información acerca de: las fuentes y la cuantía de textos académicos asignados y comprensión lectora de los textos académicos en el ámbito universitario; $\mathrm{y}$, en segundo lugar, a las estrategias que recurren los estudiantes para adentrase en tales exigencias.

Desde el punto de vista metodológico el proyecto se estructuró a partir de los planteamientos de la etnografía educativa como una disciplina que, en este caso particular, procura caminos para hacer evidente la forma como un grupo de estudiantes de la Facultad de Educación asume el control, la supervisión y la evaluación permanentes de su propio proceso de comprensión de textos académicos. las estrategias señaladas para asumir su lectura; la exposición de estrategias para la solución de dificultades encontradas ante la comprensión de los textos y las concepciones de los docentes del proceso de lectura con fines académicos; y las estrategias y frecuencias para la evaluación de la comprensión lectora y la valoración de la autonomía de los estudiantes.

El diario de campo como un instrumento principal de la observación no participante permitió acercarse a particulares formas de poner en práctica las estrategias cognitivas y metacognitivas de 
comprensión de textos académicos en ambientes de clase. Este instrumento se aplicó en tres sesiones de tres asignaturas distintas, correspondientes a los denominados "núcleos comunes" de los dos primeros semestres de las licenciaturas de la Facultad de Educación ${ }^{1}$.

La entrevista estructurada se tomó como instrumento para la recolección de información en cuanto a los mecanismos cognitivos que reconocen los estudiantes en la construcción de significado y creación de sentido al momento de enfrentar textos de carácter académico.

La población. De acuerdo con lo expuesto en el proyecto de investigación, la pregunta problema se abordó en el marco de la investigación cualitativa: el diseño evolucionó con el proyecto. De ahí que las decisiones respecto de la muestra se configuraron en el proceso mismo en un intento por acercarse y reconocer los diversos puntos de vista de los posibles participantes. Por tanto, la información guió el muestreo y evolucionó en el propio campo en aras de cubrir los requerimientos conceptuales, más que adaptarse a unas reglas metodológicas.

Ahora bien, se ha de recordar que en los estudios cualitativos casi siempre se emplean muestras pe-

1 Los "núcleos comunes" hacen referencia a una concepción curricular fundamentada en la integración deáreas del saber a través de la oferta de asignaturas comunes para los estudiantes de las cuatro licenciaturas pertenecientes al Departamento de Psicopedagogía de la Facultad de Educación de la Universidad Pedagógica Nacional. queñas. Respecto del tamaño no se pueden referir criterios o reglas inamovibles. En este caso se recurrió a un muestreo aleatorio estratificado: estudiantes que estuviesen cursando uno de los tres primeros semestres de las licenciaturas de la Facultad de Educación de la Universidad Pedagógica Nacional y profesores que, de igual manera, tuviesen a su cargo espacios académicos correspondientes a estos semestres, en particular a los denominados núcleos comunes.

\section{De las interrelaciones entre planteamientos teóricos y prácticas lectoras: los primeros hallazgos}

Para el análisis de las encuestas y de los diarios de campo se realizó una matriz a partir de dos elementos: etapas metacognitivas -planeación, supervisión y evaluación- y focos de análisis - tres aspectos que concentraron las preguntas realizadas y los eventos observados: guías para asumir los procesos de lectura, pautas de evaluación de la comprensión y procesos de lectura independiente-. A su vez, los focos se desglosaron: el primero en origen y cantidad de textos asignados y orientaciones formuladas por los docentes; el segundo, en estrategias implementadas por los docentes; y el tercero, en estrategias utilizadas por los estudiantes y su grado de autonomía, en tanto como se reiterará más adelante, se reconoce al estudiante universitario como responsable de su propio aprendizaje; es decir, como lector autónomo.

\begin{tabular}{|l|l|l|l|l|}
\hline \multicolumn{2}{|l|}{ Focos de análisis } & \multicolumn{2}{l|}{ Etapas metacognitivas } \\
\cline { 2 - 5 } \multirow{2}{*}{$\begin{array}{l}\text { Guías para asumir los } \\
\text { procesos de lectura }\end{array}$} & $\begin{array}{l}\text { Origen y cantidad de textos } \\
\text { asignados }\end{array}$ & & Supervisión & Evaluación \\
\cline { 2 - 5 } & $\begin{array}{l}\text { Orientaciones formuladas } \\
\text { por los docentes }\end{array}$ & & & \\
\hline \multirow{2}{*}{$\begin{array}{l}\text { Pautas de evaluación de la } \\
\text { comprensión }\end{array}$} & $\begin{array}{l}\text { Estrategias implementadas } \\
\text { por los docentes }\end{array}$ & & & \\
\hline \multirow{2}{*}{$\begin{array}{l}\text { Procesos de lectura } \\
\text { independiente }\end{array}$} & $\begin{array}{l}\text { Estrategias utilizadas } \\
\text { por los estudiantes }\end{array}$ & & & \\
\cline { 2 - 5 } & Autonomía & & & \\
\hline
\end{tabular}


Origen de los documentos. El ingreso a la educación universitaria presupone un ejercicio activo del estudiante como lector acucioso. Aspecto que se verá reforzado con la asignación por parte del profesor de las lecturas necesarias en el proceso de formación profesional. En el caso estudiado, se ha encontrado que al estudiante de los primeros semestres de los pregrados de la Facultad de Educación se le asignan, en promedio, de uno a seis textos por semana. La relación entre la cuantía de textos asignados por un profesor y los que realmente leen los estudiantes pareciera ser directamente proporcional. Los estudiantes encuestados se asumen como lectores activos: en su gran mayoría reconocen leer todas las lecturas asignadas; pocos afirman leer solo parte de lo ordenado.

Por lo general, el conocimiento institucionalizado circulante en las aulas universitarias proviene de fuentes igualmente institucionalizadas. En efecto, la encuesta permite inferir que un menor porcentaje de los documentos asignados proviene de la Internet. Los libros y los capítulos de libros se constituyen en la mayor fuente empleada por los docentes y sólo algunos profesores acuden a revistas o a capítulos de revistas como fundamento de la información. Al contrastar el promedio de asignación de textos por semana con la cuantía según la fuente, se evidencia una inconsistencia: la pregunta control demuestra que no es tan elevado el número de textos asignado.

Orientaciones de los profesores. Si se reconoce que el estudiante universitario debe asumirse como lector adulto, el profesor acudirá a los mecanismos que permitan generar o favorecer aquella formación. En este sentido, resultan importantes las estrategias de lectura que sugieran los profesores como vía para asumir los textos universitarios. Al respecto, la encuesta permite reconocer cuatro elementos importantes: en primer lugar, el peso que le dan los profesores a la realización de mapas y de búsqueda de ideas principales como "trucos" para afrontar la lectura; en segundo momento, se evidencia una confusión entre las instrucciones para asumir los procesos y la elaboración de documentos -se equipara extraer ideas principales con la realización de informes o reseñas-; en tercera instancia, la poca relevancia que se le da al acercamiento al contexto -búsqueda de biografías, datos históricos, por ejemplo- como posibilidad de entablar una relación de sentido; y en cuarto lugar, atender a las condiciones ambientales y ergonómicas propicias -lo confortable de "descansar diez minutos", "no leer luego de haber comido", "leer en sitio tranquilo y con calma", "hacer pausas"- como "mecanismo" de lectura; de este modo, se asemeja el acto físico con el trabajo intelectual.
Estrategias para acercarse a la comprensión lectora. En el proceso educativo se han hecho inseparables la formación y la evaluación. Ahora bien, los textos académicos se constituyen en una opción de formación en tanto que su asignación busca entregar elementos conceptuales que permitan un diálogo académico profesor-estudiante universitario. De ahí que el profesor ha de corroborar el grado de comprensión para abrir este diálogo. Sin embargo, tal ideal se ha reducido a la calificación o al "activismo".

Para el caso de la problemática estudiada, diversas estrategias permiten a los profesores evidenciar el acercamiento de los estudiantes a los textos. Como formas de comprobación, en la misma medida se solicitan estructuras de representación textual (síntesis, mapas, esquemas, etc.), elaboración de textos (resúmenes, ensayos, reseñas), resolución de pruebas orales y escritas o realización de conversatorios. Además de la nota cuantitativa, los profesores demandan ejercicios de socialización como técnicas grupales y exposiciones individuales. Solo un caso hace referencia al estudio de caso como una manera particular de probar la comprensión.

Este tipo de estrategias, según Santiago, Castillo y Ruiz (2005), han sido utilizadas principalmente como un recurso que evidencia, por una parte, el proceso lector y, por otra, los niveles de procesamiento de la información por medio de la escritura. Sin embargo, lo descrito hasta este momento no permite dar cuenta de hasta qué punto o medida los profesores utilizan este tipo de estrategias para identificar problemas de lectura y producción escrita o para determinar si les permite a los estudiantes mejorar en sus procesos de producción y comprensión.

Estrategias empleadas por los estudiantes. De acuerdo con Garner (1987b), el buen lector se caracteriza por la forma como se relaciona e interactúa con el texto: tiene conciencia y control de sus actividades cognitivas y metacognitivas durante el proceso y, además, hace uso flexible de estrategias para ajustarlas a su propósito de lectura, a la tarea(s) específica(s) y al tipo de texto. Al respecto, los estudiantes reconocen la necesidad de generar derroteros y acciones previos al acto de leer. Así, según lo deducido de las encuestas, consideran en un lugar preponderante el establecimiento de un propósito; en orden descendente de importancia, se preguntan qué saben del texto, hacen predicciones acerca del contenido, inician un proceso de prelectura, retoman las instrucciones del profesor y establecen un plan de lectura. Sin embargo, tal necesidad se quedaría en la enunciación, pues al preguntárseles por las acciones adelantadas durante 
el proceso de la lectura, ningún encuestado acude a la comprobación de lo planificado.

La autonomía de los estudiantes. Las etapas metacognitivas se hallan determinadas en gran medida por la autonomía del lector, en tanto la construcción de sentido supone su papel activo: sus lecturas irían más allá de la "tarea asignada". En consecuencia, el estudiante universitario como responsable de su propio aprendizaje se ha de reconocer como lector autónomo. Las encuestas permitieron encontrar que la mayoría de estudiantes acepta la importancia de tomar por su propia cuenta otros documentos para complementar o comprender las lecturas asignadas; esta sería una señal de relativa autonomía.

La mayoría de encuestados valora como bueno su nivel de comprensión lectora; los restantes la califican entre aceptable y regular. Ninguno reconoce tener un nivel excelente o nulo. Las razones varían entre la facilidad para comprender y la capacidad que se tiene para consultar otras fuentes; asimismo, señalan como aspectos por mejorar: la carencia de bagaje académico, una mejor actitud ante la poca "amenidad" del texto y el dominio de estrategias. Quienes otorgan una valoración de regular reconocen lo fugaz de la comprensión o atribuyen sus deficiencias a los actos docentes.

De igual manera, la mayoría considera que ha adquirido nuevas técnicas de comprensión de lectura de textos académicos mediante las asignaturas cursadas porque han tenido acceso a técnicas de comprensión lectora -ideas principales, preguntas, mapas mentales- y al señalamiento de las condiciones propicias -descansos oportunos, luz adecuada, etc.- Quienes consideraron que no han adquirido nuevas técnicas presentaron como justificación la falta de un proceso, la carencia de bases o el hecho de poseer ya dichas técnicas.

Puede afirmarse que no existe una marcada diferencia entre quienes consideran que poseen estrategias para comprender los textos que, como universitarios, deben leer y quienes no lo reconocen. El hecho de que aún les falta experiencia, de que requieren llevar un proceso y de que necesitan leer más o tener más referentes se encuentran dentro de las razones por las cuales los estudiantes consideran que no poseen las estrategias adecuadas para asumir los textos académicos. Quienes consideran que aún no poseen las estrategias suficientes mencionan una variedad de faltas: de lectura, de rigurosidad, de experiencia, de herramientas y de reconocimiento del papel de lo afectivo como determinante en este proceso, entre otras.
La encuesta dirigida a los profesores. Las respuestas otorgadas por los profesores respecto del origen de los documentos asignados para leer reafirman las apreciaciones de los estudiantes: se tornan mínimas las lecturas provenientes de la Internet y, por el contrario, predominan las fuentes institucionalizadas: libros o capítulos de libros.

En relación con las estrategias formuladas para hacer evidente el acercamiento de los estudiantes a los textos académicos, existe coincidencia entre las respuestas otorgadas por los estudiantes y las entregadas por los profesores: se enfatiza en las síntesis, en los resúmenes y en los conversatorios. De igual manera, no se hace evidente el acercamiento al contexto como posibilidad de vía hacia la resignificación de lo leído.

Al momento de realizar controles de lectura, los profesores dan mayor peso cuantitativo al análisis y a la interpretación; la memorización no tiene mayor trascendencia. De otra parte, la socialización se constituye, según los profesores, en la principal estrategia no cuantitativa para comprobar la comprensión de lectura.

En cuanto a las acciones previas a la lectura, profesores y estudiantes coinciden en valorar de manera suma el establecimiento de un propósito para la lectura y el preguntarse qué se sabe acerca del texto o del tema. Los estudiantes dan una mediana valía a la realización de predicciones acerca del contenido $\mathrm{y}$ al inicio de un proceso de prelectura, aspectos que no tienen relevancia para los profesores. Por su parte, los profesores consideran medianamente importante establecer un plan de lectura y retomar las instrucciones que han dado a sus estudiantes como estrategias para afrontar textos de carácter académico.

Aun cuando pocos estudiantes señalaron acudir a sus profesores al momento de encontrar dificultades en la comprensión, los profesores reconocieron poseer algunas estrategias para brindar orientaciones al respecto: señalar hipótesis para que se confronten con el texto, tratar de identificar la fuente de las dificultades, sugerir lecturas alternas e invitar a seguir leyendo para buscar más información.

Mientras que la mayoría de los estudiantes valora como bueno su nivel de comprensión lectora, los profesores les atribuyen apenas un aceptable. Aluden razones que giran alrededor de las dificultades y del déficit: dificultades en los procesos de comprensión tanto macro y micro, carecimiento de estrategias claras para abordar un texto universitario, insuficiencia en el análisis y en la interpretación, tendencia a no superar la lectura literal. 
La mayoría de profesores encuestados considera que los estudiantes de los primeros semestres de pregrado no poseen estrategias de comprensión lectora adecuadas para afrontar los textos que, como universitarios, deben leer; manifiestan tres tipos de razones: las carencias de los estudiantes -leen de forma mecánica, carecen de interpretación y argumentación, temen a la dificultad de los textos; sin embargo, muestran interés por implementar nuevas estrategias-, la complejidad del proceso universitario -en el primer semestre se les dificulta abordar este tipo de textos; requieren continuar en el proceso en consonancia con otros espacios académicos y con otros niveles de mayor exigencia-y deficiencias del sistema educativo -la primaria y la secundaria no proporcionan a los estudiantes estrategias de comprensión lectora-.

Los profesores recomiendan unos aspectos mínimos que favorecería el proceso lector en tanto que acto metacognitivo: para la etapa de planeación, sugieren la prelectura, la identificación de conocimientos previos y el establecimiento de propósitos de lectura; para la etapa de supervisión, realizar esquemas, mapas conceptuales, glosarios, lecturas complementarias y conversatorios y plantearle preguntas al texto. De particular interés sería anotar el hecho de que en las sugerencias no hay cabida a la etapa de evaluación. Al respecto, se ha de recordar que la evaluación va ligada a la posibilidad de plantear cambios. Si aquí no se considera la evaluación, entonces ¿el actual modo de enfrentar la lectura no requiere cambios?

Finalmente, resaltar dos de los aspectos sugeridos en la etapa de supervisión: la reflexión con base en el análisis del texto alrededor de la profesión y la ubicación del texto en un contexto con los autores citados y los términos específicos de cada disciplina. Llama la atención el hecho de que los profesores lo hayan expresado al final de la encuesta en el espacio de las sugerencias, pero que no lo hayan incluido en el momento en que se les preguntó por otro tipo de actividades que podrían realizar para comprobar el acercamiento a las lecturas por parte de los estudiantes.

La entrevista. Su análisis permitió establecer cinco categorías:

a. La comprensión como: entender -"es la interpretación clara", "El comprender conceptos", la "capacidad de entendimiento"-, dar cuenta -"expresar con mis propias palabras", "hacer un escrito, apropiarme del tema"- y encontrar ideas principales -"sacar mis propias ideas", "recordar aspectos importantes"-. b. Las relaciones al interior de un texto comprendido: del texto -"pues el texto debe darme una serie de contenidos, que de una o de otra forma al seguir el texto detenidamente, debe dar cuenta de una secuencia de significados y lo que se ha comprendido previamente" -, del lector -"cuando de lo más mínimo o la palabra más explícita saco mi conclusión"-, de la epistemología -"lo identifica con conocimientos anteriores ya trabajados"y con el exterior -"al comprender un texto se puede hacer relación con situaciones ya vividas o similares a las cotidianas"-.

c. La relación entre un texto comprendido y otros textos: comparar - "tener contraposiciones" y establecer coincidencias entre sus teorías y/o afirmaciones- y trabajar conceptos - "llegar a un concepto más claro y complejo", "explicar contenidos".

d. Modificación del desempeño académico para la comprensión de un texto: dar cuenta del texto -resaltar "aspectos importantes", "comprender texto presentes y futuros"-, modificar actos y formas de pensar -"no solo leer por leer" y sentir "segura de explicar"- y adquirir conocimientos y herramientas -"Adquirir conocimientos para su formación y nuevo saber"-.

e. La determinación del sentido de una lectura permite reconocer una interrelación de las categorías emergentes: la toma de sentido de la lectura pasa por lo afectivo, lo cognitivo y lo social: "relaciono con la experiencia de momento sino con experiencias a futuro", "gusto poder argumentar, profundizar", "llena de conocimientos para mí como persona” y "adquiero una posición de crítica ante algo que manejo plenamente".

Los diarios de campo. Su estudio permitió establecer una serie de actividades ordenadas o sugeridas por los profesores que pueden relacionarse con las etapas de la metacognición como base de procesos autónomos y conscientes del trabajo académico.

En relación con la etapa de planeación, se encontró en una de las asignaturas la construcción de un documento permanente como estrategia para asumir las lecturas de textos durante un semestre. La glosa se asigna como un ejercicio de pasos consecuentes que van desde la identificación de palabras, frases o términos que pueden ocasionar dificultades en la comprensión; pasa por la consulta de diferentes fuentes para complementar los conceptos que no fueran claros para el lector; preguntarse por algunos aspectos biográficos del autor, lugar, época y tensiones del momento histórico en el que se escribió el texto; y llega a la construcción particular de conceptos.

Respecto de la etapa de supervisión, se observó que, aun cuando no era frecuente, se invitaba al planteamiento de preguntas para acercarse al proceso de comprensión. De igual manera, se observaron casos en donde se realizaba un detenimiento en aspectos 
gramaticales, no por la gramática misma sino como elementos articuladores de comprensión. En otras instancias, se generaron preguntas para orientar la comprensión, otras para verificar la lectura y la comprensión y otros plantean preguntas con el fin de establecer relaciones de comprensión entre distintos textos.

En cuanto a la etapa de evaluación, por lo general se tomaron producciones escritas de los estudiantes $y$, a partir de esos errores, se señalaron los problemas de comprensión que van desde lo conceptual hasta el seguimiento de ciertas estructuras y estilos que de no entenderse o reconocerse terminan por afectar la comprensión.

\section{De las conclusiones del estudio}

Este acercamiento hace referencia a un acto de cierre para una apertura. Más que un punto de llegada, confluye en un lugar de partida: expresa interrogantes derivados de la riqueza de las voces de los estudiantes y de los profesores que se confrontan y se enfrentan en actos lectores y en comprensiones de lectura. En últimas, cada intento de investigación termina por configurar exploraciones que abren caminos para otras indagaciones.

Indagar acerca de las estrategias cognitivas y metacognitivas en la comprensión de textos significa reconocer los procesos lectores y su relación con el actuar de los como estudiantes universitarios y, a su vez, pensar formas de construir y reconstruir actos de enseñanza que, por supuesto, sugerirían conexiones con perspectivas pedagógicas y propuestas curriculares concretas. Sin embargo, acercarse a tales instancias demanda un proceso que puede iniciarse en las respuestas que aquí se brinden. Vale la pena al respecto señalar que tales respuestas no aluden a una pretensión de "última palabra". Se pueden tomar como una mirada frente a interrogantes particulares de las investigaciones adelantadas, pero generales en cuanto a la lectura en formación universitaria: al momento de leer textos académicos, ¿existe un propósito de lectura?, ¿se sigue un plan?, ¿el lector actúa de modo intencional, autónomo, independiente y responsable de su propio aprendizaje? En los procesos universitarios de formación docente, ¿se encuentran estudiantes que regulen sus propios procesos de lectura y, a su vez, profesores que propicien esta transformación?, ¿se consolidan allí ambientes donde los procesos académicos generen estrategias de comprensión? En fin, ¿hasta dónde se propician procesos interactivos, intencionales, constructivos e inferenciales en los procesos académicos de formación de docentes?
Respecto de estos interrogantes, se pueden enunciar algunos hallazgos del estudio:

- Se reitera en la importancia de la comprensión de lectura; pero, en pocas ocasiones, se establecen relaciones con la generación de estrategias para comprender lecturas. Asimismo, se plantean aproximaciones al trabajo con los textos, se proponen algunas estrategias para trabajarlos, se asignan tareas, pero no se realizan actividades de supervisión: qué se comprendió de la tarea, cómo se realizó, para qué, de qué manera. La realización de la tarea se constata y se califica pero no se retoma en relación con aciertos y fallas para traer a colación estrategias para posibilitar y facilitar nuevas comprensiones. En otras palabras, se asimila la tarea como sinónimo de comprensión: asignada la tarea, comprendida la lectura; tarea puesta, comprensión dada.

- Si bien los profesores recurren a algunos elementos para mejorar la comprensión lectora, no se establece si se preguntan por la pertinencia de cada estrategia en particular ni por su relación o coherencia con cada temática, actividad o forma de abordar la enseñanza. Tampoco se señala si las estrategias empleadas por los estudiantes se consideran como las más apropiadas para abordar determinados textos o temáticas. En general, los eventos de clase ratifican la importancia de la comprensión lectora, pero en pocas ocasiones generan estrategias de comprensión.

"Se reitera en la importancia de la comprensión de lectura; pero, en pocas ocasiones, se establecen relaciones con la generación de estrategias para comprender lecturas. Asimismo, se plantean aproximaciones al trabajo con los textos, se proponen algunas estrategias para trabajarlos, se asignan tareas, pero no se realizan actividades de supervisión: qué se comprendió de la tarea, cómo se realizó, para qué, de qué manera." 
- Frente a la guía o el apoyo del profesor, algunos estudiantes manifiestan el poco énfasis dado a la planeación y a la supervisión de su proceso lector. La supervisión pareciera quedarse en la calificación, en la nota numérica. En ocasiones, además, se califica el proceso de comprensión como válido si el estudiante dice del texto aquello que el profesor quiere que se diga y de la manera como quiere que se diga. La tarea se torna castigo; no se toma como forma de comprensión o de crecimiento sino como ocupación: un estudiante "lleno de tareas", aunque no las comprenda.

- Ante la evidencia de falta de comprensión lectora del estudiante no se genera un proceso de estrategias o de posibilidades de acercamiento textual sino que se tiende a la adjetivación personal. Es decir, se deja de lado la pregunta por la comprensión o por las estrategias y se confronta la respuesta del individuo; en esta confrontación se tiende a calificar y a adjetivar, se "regaña" al estudiante según sus acciones.

- La constatación de las tareas tendería hacia la calificación y no a la evaluación, pues no se retoman los niveles de las fallas de comprensión para proponer otras formas de acercamientos a las lecturas. El estudiante considera finalizado el proceso con la nota asignada; el profesor prepara e indica una nueva tarea. Se plantean aproximaciones al trabajo con los textos, se asignan tareas, pero no se proponen actividades de construcción y deconstrucción de esas tareas.

- Por otra parte, los esquemas de representación textual -mapas, cuadros, estructuras de síntesis, etc.- concentran, en gran medida, las maneras de dar cuenta de la comprensión de un texto. Cuando se solicitan documentos como ensayos, reseñas o resúmenes o exposiciones de orden oral como conversatorios, debates o seminario no se especifica con claridad en qué se diferencian los tipos de textos, las diversas formas de exposición, ni qué significado o sentido tiene solicitarlos como tareas que dan cuenta de procesos de comprensión.

- Para los estudiantes configurar un plan de lectura ante las exigencias de comprensión lectora de los textos académicos se torna primordial. Pero tal enunciación no encuentra lugar, pues no se acude a su comprobación ni al establecimiento de otro plan ni a continuar o a seguir aquel que ha sido pertinente.

- Llama la atención la tendencia a considerar todavía como sinónimo de estrategias de lectura a las denominadas, hace algunas décadas, "técnicas de lectura" relativas al espacio físico donde sucede la actividad lectora: el sitio de estudio, la iluminación, el bienestar y el confort, entre otras.

- La planeación, la supervisión y la evaluación permanente, como estrategias de la metacognición, permiten la emergencia de la autonomía como proceso consciente del acto lector de textos académicos en el ámbito universitario. Sin embargo, parecen no hacerse evidentes ciertas didácticas que movilicen estas estrategias, la potenciación de la autonomía no se acercaría a lo esperado para un ámbito universitario, más aún referido a pregrados en licenciaturas. El estudiante pareciera exigir una autonomía que no quiere asumir; el profesor, más allá de orientar o de guiar, solicita tareas.

- La no cohesión entre la lectura y la generación de estrategias demanda el planteamiento de interrogantes pedagógicos. Los acercamientos a estrategias de comprensión, su supervisión y evaluación, la diferenciación de tipos de textos, sus formas de leerlos y comprenderlos tienden a quedarse en las asignaturas de producción y comprensión de textos -o de nombres similares, según la institución-y poco o nada se recuperan por parte de los profesores y de los estudiantes en otros espacios. De ahí que la lectura se constituya en problema, en tanto que tropiezo, pero todavía dista de tomarse en sus implicaciones curriculares.

"La planeación, la supervisión y la evaluación permanente, como estrategias de la metacognición, permiten la emergencia de la autonomía como proceso consciente del acto lector de textos académicos en el ámbito universitario. Sin embargo, parecen no hacerse evidentes ciertas didácticas que movilicen estas estrategias, la potenciación de la autonomía no se acercaría a lo esperado para un ámbito universitario, más aún referido a pregrados en licenciaturas."

Como cierre, una observación relevante. Páginas atrás se señaló que: "el conocimiento institucionalizado circulante en las aulas universitarias proviene de fuentes igualmente institucionalizadas"; asimismo, se reiteró que los documentos trabajados como textos académicos en los pregrados aludidos provenían 
de libros como fuentes confiables de ese saber. La reiteración encuentra su motivación ante el mínimo uso -o más bien ninguno- que hacen los docentes de artículos provenientes de revistas académicas o científicas. A su vez, el llamado se justifica en dos aspectos: en primera instancia, la exigencia por parte de una actualidad totalizadora y certificadora que demanda de los docentes (investigadores) la elaboración de cuantiosas publicaciones que alimenten la continuidad de revistas académicas; en segundo lugar, la Universidad Pedagógica Nacional , en general, y su Facultad de Educación, en particular, vienen acumulando esfuerzos en torno de unas publica-

\section{Bibliografía}

Alliende, F. (1982, marzo), “La comprensión de la lectura y su desafío”, en: Lectura y vida, año 3, núm. 1.

Baker, L. y Brown, A. L. (1984), "Metacognitive Skills and Reading”, en: Pearson, P. D., Handbook of reading research, Logman.

Beaugrande, R. A. de (1980), Text, Discourse and Process, Norwood, N J, Ablex Pub.

Beaugrande R. A. de y Dressler W. (1983), Introduction to Text Linguistics, London, Longman.

Beaugrande, R. A. de (1984), Text Production. Towards a Science of Composition, Norwood, N. J., Ablex Pub.

Bransford J. D. y Johnson M. K. (1990), “Consideraciones sobre algunos problemas de la comprensión”, en: Valle, F. y otros (ed.). Lecturas de sociolingüística, Madrid, Alianza.

Brown, A. (1980), "Metacognitive development and Reading”, en: Spiro, J.; Bruce, B. C. y Brewer, W. F. (1980, eds.)

Cañón Flórez, L. (1994), “El ensayo y el informe”, en: Revista Escuela de Administración de Negocios.

Cañón Flórez, L. (1999-2000), Comprensión de lectura. 10 niveles, Bogotá, Hispanoamérica.

Cañón Flórez, L. y Molina, C. (2004, segundo semestre), “La oralidad y la escritura: planteamientos y reflexiones en torno a la clase de español", en: Pedagogía y saberes. No. 21 , pp. 55-62.

Flavell, J. (1996), El desarrollo cognitivo, Madrid, Visor.

Flavell, J. H. (1981), Cognitive monitoring, en: Dickson, W. P. (eds.), Children's Oral Communication Skills, New York, Academic Press. ciones periódicas institucionales que lleguen a y se mantengan en los exigentes estándares del Publíndex y de otras escalas similares.

Si la producción académica de los docentes investigadores depositada en revistas no se reconoce por los docentes mismos como fuente "confiable" del saber institucionalizado, se crea un gran número de interrogantes frente a la razón misma de la escritura y de las publicaciones en la educación superior. Pareciera que, como ocurre con los trabajos de grado, cumplir con las exigencias de publicación en revistas indexadas simplemente reposará en los anaqueles de gloriosas bibliotecas a la espera de un despistado lector.

Garner, R. (1987a), "Strategies for Reading and Studying Expository Texts", en: Educational Psychologist, vol. 22, núm. 3-4, pp. 299-312.

Garner, R. (1987), Metacognition and Reading Comprehension, N.J., Ablex Publishing.

González, J. (1991), "Las inferencias durante el proceso lector", en: Puente, A. (Comp.), Comprensión de la lectura y acción docente, Madrid, Ediciones Pirámide, pp. 110-136.

Goodman, K. (1967, mayo), "Reading. A Psycholinguistic Guessing Game”, en: Journal of Reading Specialist, núm. 6, pp. 126-135.

León, J. A. (1991), “La mejora de la comprensión de lectura, un análisis interactivo", en: Infancia y aprendizaje, núm. 56, Madrid.

López, G. S. (1997), "Los esquemas como facilitadores de la comprensión y aprendizaje de textos", en: Revista Lenguaje, Cali, Facultad de Humanidades, Universidad del Valle, núm. 26.

López, G. S. (1998), La metacomprensión y la lectura, en: Martínez, M. (1998, comp.).

López, G. S. y Conover, N. (1980), “Determinación de necesidades en la enseñanza-aprendizaje de idiomas extranjeros. Informe de Investigación", en: Revista Lenguaje, Departamento de Idiomas, Universidad del Valle, núm. 11.

López, G. S. y Arciniegas, E. (2004), Metacognición, lectura y construcción de conocimiento. El papel de los sujetos en el aprendizaje significativo. Cátedra UNESCO, Cali, Editorial Universidad del Valle. 
Martínez M. C. (1994), Análisis del discurso, Cali, Editorial Universidad del Valle.

Martínez M. C. (1998), "EI desarrollo de estrategias discursivas a nivel universitario", en: Martínez, M. C. (1998, comp.).

Martínez, M. C. (1998, comp.), Los procesos de la lectura y la escritura, Cali, Editorial Universidad del Valle.

Mateos, M. (2001), Metacognición y educación, Buenos Aires, Aique.

Newell, A. (1980), "Inteligencia artificial y concepto de la mente", en: Revista Teorema.
Singer, H y Rudell, R. B. (ed.), Theoretical Models and Processes of Reading, IRA Newark, Delaware.

Santiago, A. W.; Castillo, M. C. y Ruiz, J. (2005), La lectura, metacognición y evaluación, Bogotá, Alejandría.

Smith, F. (1990), Para darle sentido a la lectura, Madrid, Visor.

Smith, F. (1971), Reading, Cambridge, Cambridge University Press.

Spiro, J.; Bruce, B. C. y Brewer, W. F. (1980, eds.), Theoretical Issues in Reading Comprehension, Hillsdale, NJ: Lawrence Erlbaum Associates 